\title{
Unstable periodic motion in turbulent flows
}

\author{
G. Kawahara ${ }^{1}$, S. Kida ${ }^{2}$, and L. van Veen ${ }^{3}$ \\ ${ }^{1}$ Department of Mechanical Science, Graduate School of Engineering Science, Osaka University, Osaka 560-8531, Japan \\ ${ }^{2}$ Department of Mechanical Engineering and Science, Graduate School of Engineering, Kyoto University, Kyoto 606-8501, \\ Japan \\ ${ }^{3}$ Department of Mathematical and Statistical Science, La Trobe University, Victoria 3086, Australia
}

Received: 18 April 2006 - Revised: 12 July 2006 - Accepted: 14 July 2006 - Published: 21 September 2006

\begin{abstract}
Recently found unstable time-periodic solutions to the incompressible Navier-Stokes equation are reviewed to discuss their relevance to plane Couette turbulence and isotropic turbulence. It is shown that the periodic motion embedded in the Couette turbulence exhibits a regeneration cycle of near-wall coherent structures, which consists of formation and breakdown of streamwise vortices and low-velocity streaks. In phase space a turbulent state wanders around the corresponding periodic orbit for most of the time, so that the root-mean-squares of velocity fluctuations of the Couette turbulence agree very well with the temporal averages of those along the periodic orbit. The Kolmogorov universal-range energy spectrum is observed for the periodic motion embedded in high-symmetric turbulence at the Taylor-microscale Reynolds number $R e_{\lambda}=67$. A laminarization strategy inspired by investigation of the phase-space structure in the vicinity of the unstable periodic orbit is presented for the Couette turbulence.
\end{abstract}

\section{Introduction}

The states of fluid motion are generally classified into laminar and turbulent flow. The turbulent flow exhibits spatiotemporally chaotic behaviour and its details repeat neither in space nor in time. Most of fluid motion seen in daily life, such as water flowing out of a fully-turned-on tap and vortical smoke emitted from a chimney, is turbulent.

We have a very long history of turbulence research since the famous experiments of the transition to turbulence in circular pipe flow performed by Reynolds (1883) over 120 years ago. In the course of the research statistical properties, e.g., mean velocity and velocity correlation, of various kinds of turbulent flows have been investigated to find out

Correspondence to: G. Kawahara

(kawahara@me.es.osaka-u.ac.jp) that there exist universal statistical laws of turbulence, such as the $-5 / 3$ power law of energy spectrum in the inertial range and the logarithmic law of mean velocity profile in wall turbulence (see Davidson, 2004). However, it is still difficult to quantitatively explain what is going on in turbulent flows and how the events relate with statistical properties of turbulence. This difficulty might arise from the fact that we do not have any simple spatiotemporal description of chaotic behaviour of turbulent flows.

Recently, much experimental and numerical evidence for the existence of striking coherent motion and structures has been found in various turbulent flows, and the coherence in turbulence has attracted attention of many investigators (see Holmes et al., 1996). Near-wall streaks and streamwise vortices, which will be described in Sects. 2 and 3, are typical examples of coherent structures. Besides them a variety of coherent structures, e.g., tubular vortices in isotropic turbulence, transverse rollers in mixing-layer turbulence, hairpin vortices and so on, have been reported. The coherent structures are much simpler than incoherent turbulence because we can identify them and track their temporal evolution. Therefore, it is expected that we would be able to understand complex spatiotemporal structures of turbulence through the coherent structures. In order to characterise the spatiotemporal structures of turbulence in terms of the coherent structures, we must first define strictly the coherence in turbulent flows with strong irregularity, where no two flow structures with completely the same shape are observed.

In the present paper we review a new approach to the problem of defining coherent structures in turbulent flows. In this approach the incomplete coherence appearing in autonomous turbulent systems is exactly characterised by unstable periodic solutions to the Navier-Stokes equation. This paper is organised as follows. In Sect. 2 we discuss the recurrent dynamics of coherent structures found by Jiménez and Moin (1991) and Hamilton et al. (1995) in near-wall turbulence, which is the trigger of the course of our research. Motivated

Published by Copernicus GmbH on behalf of the European Geosciences Union and the American Geophysical Union. 
by these findings, in Sect. 3 we obtain unstable periodic motion numerically in plane Couette turbulence, which is one of canonical near-wall turbulence, and we describe exactly the recurrent behaviour of coherent structures in terms of the periodic motion (Kawahara and Kida, 2001). In Sect. 4 the recently found unstable periodic motion representing isotropic turbulence is reviewed (van Veen et al., 2006), and in Sect. 5 turbulence control strategy inspired by unstable periodic motion is briefly explained (Kawahara, 2005). Finally, concluding remarks are given in Sect. 6.

In this paper the approach by unstable periodic orbits will not be applied to geophysical problems; however, we expect that the present approarch would also be useful in geophysics. Actually, Kazantsev (2001) has recently described deterministic chaos in the barotropic ocean model in terms of unstable periodic orbits.

\section{Regeneration cycle in near-wall turbulence: the trig- ger of the course of research}

There exist two kinds of coherent structures in the near-wall region of turbulent flows. One is the low-velocity streak which is elongated in the streamwise direction and arranged roughly periodically in the spanwise direction. The other is the streamwise vortex which takes a tubular form with its rotation axis aligning with the streamwise direction. The streamwise vortices often appear along the streaks. In nearwall turbulence the streaks and the vortices exhibit complicated behaviour in space and time.

In their direct numerical simulations of plane Poiseuille turbulence, which is pressure-driven turbulent flow between two parallel plates, Jiménez and Moin (1991) minimized the streamwise and spanwise dimensions of a computational periodic box while sustaining turbulence activity to obtain the simplified turbulent field without any irregular largescale motion. In this numerically established turbulence the streaks and vortices arrange periodically in the wall-parallel directions. As a consequence of reducing spatial complexity of turbulence, they found cyclic dyamics for the regeneration of coherent structures embedded in turbulent motion. By using the same numerical technique, Hamilton et al. (1995) lowered the Reynolds number to realize highly regularized plane Couette turbulence, which is driven by two parallel plates moving with different constant velocities. In this plane Couette turbulence with high spatial coherence they reported a recurrent dynamical process, that is, regeneration cycle (or temporal periodicity), of formation and breakdown of the near-wall streamwise vortices and the low-velocity streaks, in a qualitative way.

In chaotic systems of few degrees of freedom, such as the Hénon map (Auerbach et al., 1987) and the Lorenz system (Franceschini et al., 1993), a number of unstable periodic orbits, which are embedded in a chaotic attractor, have been shown to provide a useful characterisation of proper- ties of the attractor. Recently the existence of a periodic solution to a dissipative partial differential equation (namely the Kuramoto-Sivashinsky equation) has been proved by a rigorous computer-assisted method (Zgliczyński, 2004), and spatiotemporal chaos appearing as a solution to the partial differential equation has been characterised in terms of unstable periodic orbits (Christiansen et al., 1997; Zoldi and Greenside, 1998). In this context, it is expected that the simplest description, in phase space, of spatiotemporal coherence in turbulence should be given by an unstable periodic orbit embedded in a turbulent attractor. Although it is much more difficult to find an unstable periodic orbit in that highdimensional system, there is a possibility of extracting a periodic orbit in the above-mentioned highly regularized plane Couette turbulence of relatively low degrees of freedom. The recurrent behaviour found by Hamilton et al. (1995) suggests the existence of unstable periodic motion embedded in the Couette turbulence.

\section{Unstable periodic motion in plane Couette turbulence}

In this section we consider the Couette turbulence under the same conditions as those investigated by Hamilton et al. (1995) to search for the periodic solution (Kawahara and Kida, 2001). We first perform direct numerical simulations of the incompressible Navier-Stokes equation, by using a spectral method, for the plane Couette turbulence. The simulation code used in this work was developed by Toh (see Itano and Toh, 2001). The time-advancement is achieved by the 2nd-order Adams-Bashforth scheme for the nonlinear terms and the Crank-Nicolson scheme for the viscous terms (Kim et al., 1987). The geometry of plane Couette flow is shown in Fig. 1. The upper and lower plates at $y= \pm h$ move in the streamwise $(x)$ direction at constant velocities $\pm U$, respectively. We impose no-slip boundary conditions on the plates where the fluid velocity coincides with the plate velocity. A laminar state, which is represented by a linear profile $U y / h$ of the streamwise velocity, in the plane Couette system is known to be linearly stable at any Reynolds number, implying that the laminar state has a basin of attraction in phase space. Therefore the state tends to laminar or turbulent flow depending on the initial condition. The developed turbulent states are obtained from the long-time simulations from appropriate initial conditions.

The flow is supposed to be doubly periodic with the spatial periods, $L_{x}$ and $L_{z}$, in the wall-parallel directions, $x$ and $z$. The volume flux in the $x$-direction and the mean pressure gradient in the $z$-direction are set to be zero. The streamwise and spanwise periods are $\left(L_{x}, L_{z}\right)=(1.755 \pi, 1.2 \pi)$ (Hamilton et al., 1995). The dealiased Fourier expansions are employed in the streamwise and spanwise $(z)$ directions, and the Chebyshev-polynomial expansion in the wall-normal $(y)$ direction. Numerical computations are carried out on 8,448 $(=16 \times 33 \times 16$ in $x, y$, and $z)$ grid points at $R e \equiv U h / \nu=400$, 


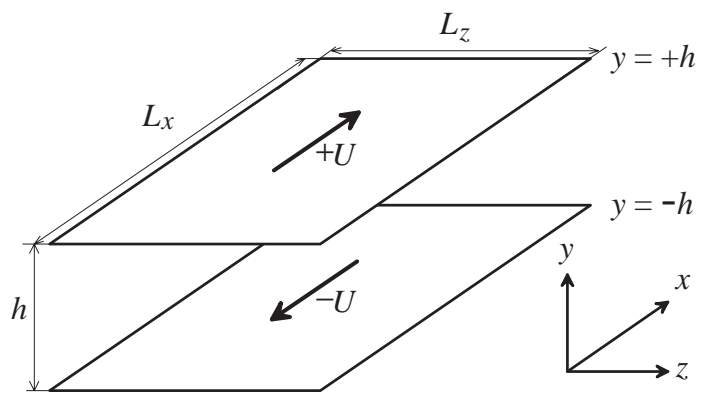

Fig. 1. Couette flow configuration.

where $v$ is the kinematic viscosity of fluid. It is known that the mean velocity profile in the near-wall region of turbulent flows scales with $v$ and the wall friction velocity $u_{\tau}=\sqrt{\tau_{w} / \rho}$, being independent of the Reynolds number and the way of driving the flow (see Davidson, 2004). Here $\tau_{w}$ is the mean wall shear stress, and $\rho$ is the mass density of the fluid. Hereafter $(\cdot)^{+}$denotes the quantity non-dimensionalised by $v$ and $u_{\tau}$. The sizes normalised by $v / u_{\tau}$, of the computational periodic box are $\left(L_{x}^{+}, 2 h^{+}, L_{z}^{+}\right)=(188,68,128)$.

The energy is injected through the frictional force on the moving walls and consumed at small scales over the whole flow field by viscous dissipation. The energy input rate

$$
I=\frac{h}{2 L_{x} L_{z} U} \int_{0}^{L_{x}} \int_{0}^{L_{z}}\left(\left.\frac{\partial u}{\partial y}\right|_{y=-h}+\left.\frac{\partial u}{\partial y}\right|_{y=+h}\right) \mathrm{d} x \mathrm{~d} z
$$

and dissipation rate

$D=\frac{h}{2 L_{x} L_{z} U^{2}} \int_{0}^{L_{x}} \int_{-h}^{+h} \int_{0}^{L_{z}}|\omega|^{2} \mathrm{~d} x \mathrm{~d} y \mathrm{~d} z$

normalised by those for a laminar state are calculated, where $u$ is the streamwise velocity and $\omega$ is the vorticity vector. The energy input and dissipation vary in a chaotic way in time, and their temporal averages, which are substantially larger than the corresponding ones $(I=D=1)$ in a laminar state are the same $(I=D \approx 3)$ because the turbulence is statistically stationary.

In the present numerical scheme the independent variables are 31 Chebyshev coefficients for the mean streamwise and spanwise components of velocity, $7424(=16 \times 29 \times 16)$ Fourier-Chebyshev-Fourier coefficients for the wall-normal velocity, and $7936 \quad(=16 \times 31 \times 16)$ Fourier-ChebyshevFourier coefficients for the wall-normal vorticity. The number $N$ of degrees of freedom of the present dynamical system is therefore about 15000. An instantaneous state of the flow field and its temporal evolution should be represented respectively as a point and its trajectory in the $N$-dimensional phase space spanned by all the independent variables. In Fig. 2, we plot, with a solid line, a projection of the turbulence trajectory over a period of $10^{4} \mathrm{~h} / U$ on the two-dimensional subspace spanned by $I$ and $D$. Grey dots are attached at intervals of $2 h / U$. The orbit generally tends to turn clockwise.

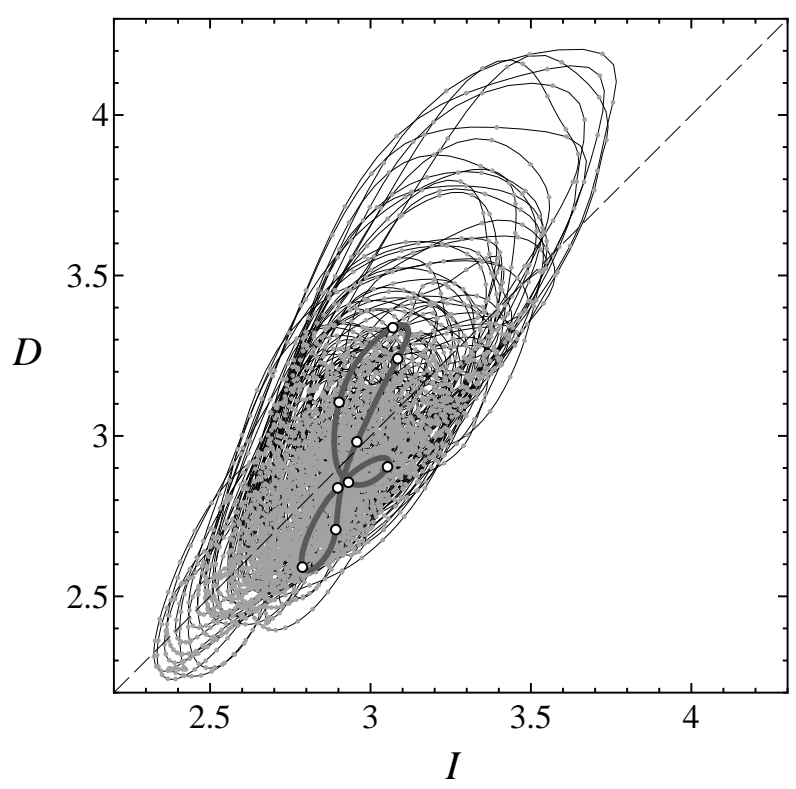

Fig. 2. Projections of a turbulent and a periodic orbit on the $(I, D)$ plane (Kawahara and Kida, 2001). The lateral and longitudinal axes respectively represent total energy input rate $I$ and dissipation rate $D$ normalised by those for a laminar state. The solid line denotes the turbulence trajectory, to which grey dots are attached at intervals of $2 h / U$. A closed grey line denotes a periodic orbit. Nine circles on the periodic orbit indicate the phases of panels (a-i) in Fig. 3. The energy input and dissipation rates are in balance on the dashed diagonal.

The energy input and dissipation rates are in balance on the dashed diagonal. Though Hamilton et al. (1995) reported the recurrent behaviour of the Couette turbulence, the variation of the trajectory seems to be in a chaotic state rather than in a periodic state.

Now we shall search for a periodic orbit embedded in the turbulence attractor. In a turbulent state, the spatial symmetries in the Nagata (1990) steady solution:

(i) the reflection with respect to the plane of $z=0$ and a streamwise shift by a half period $L_{x} / 2$, and

(ii) the $180^{\circ}$ rotation around the line $x=y=0$ and a spanwise shift by a half period $L_{z} / 2$,

are observed to appear approximately without being imposed on the flow. Therefore, we impose them on a time-periodic solution to be searched for, which allows us to reduce the degrees of freedom roughly to $N / 4$. Note that a solution with these symmetries cannot be a travelling wave in either the $x$ - or the $z$-direction.

In the phase space we take a Poincaré section $\operatorname{Im}\left(\widetilde{\omega}_{y 0,0,1}\right)=-0.1875 U / h$ to define a Poincaré map, where $\operatorname{Im}\left(\widetilde{\omega}_{y 0,0,1}\right)$ is the imaginary part of the Fourier-ChebyshevFourier coefficient of the wall-normal vorticity for the zero 


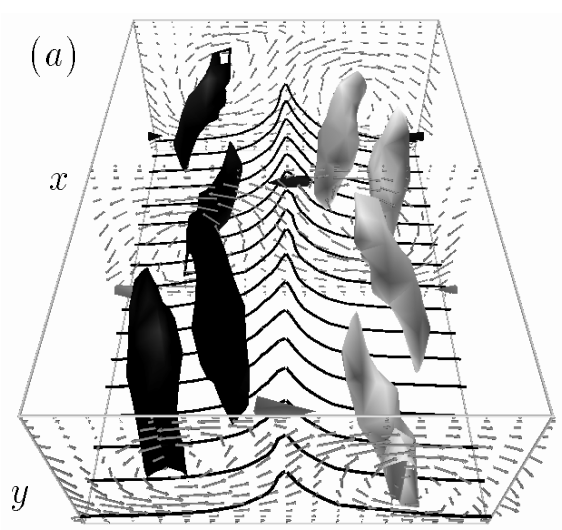

$z$

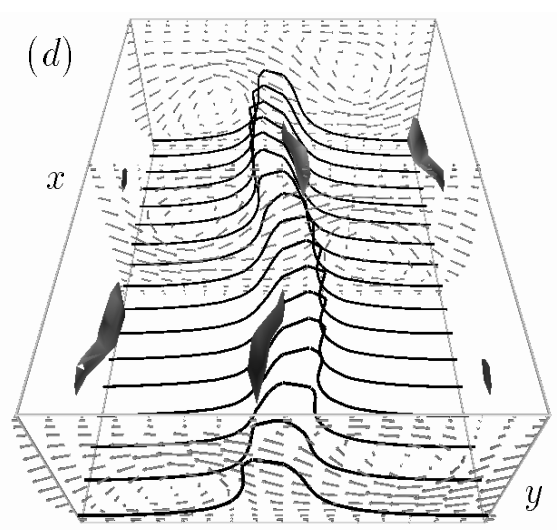

$z$

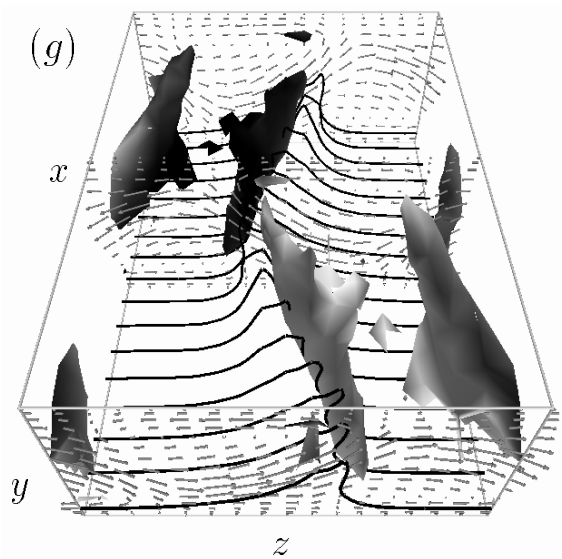

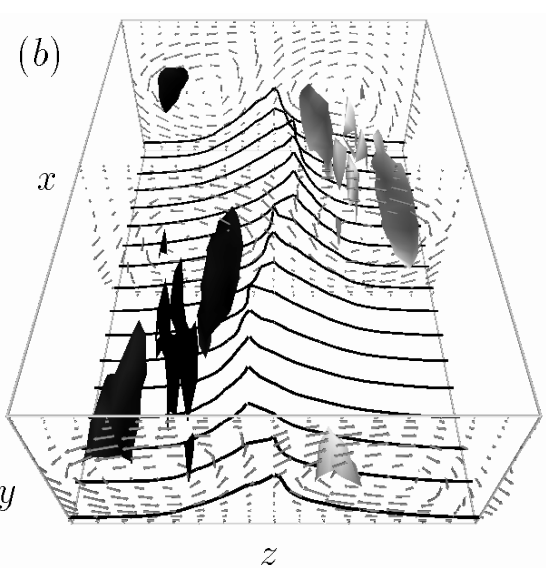

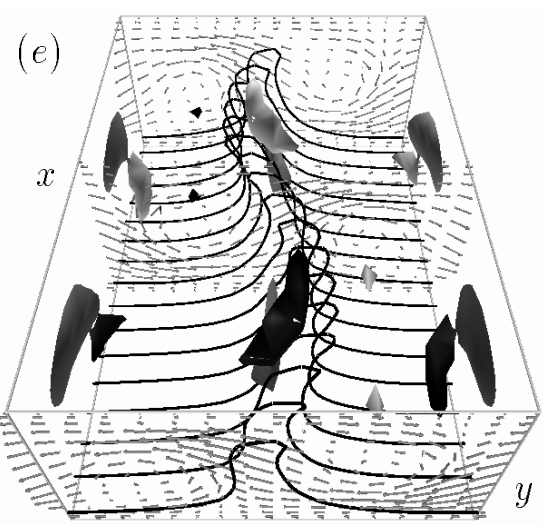

$z$

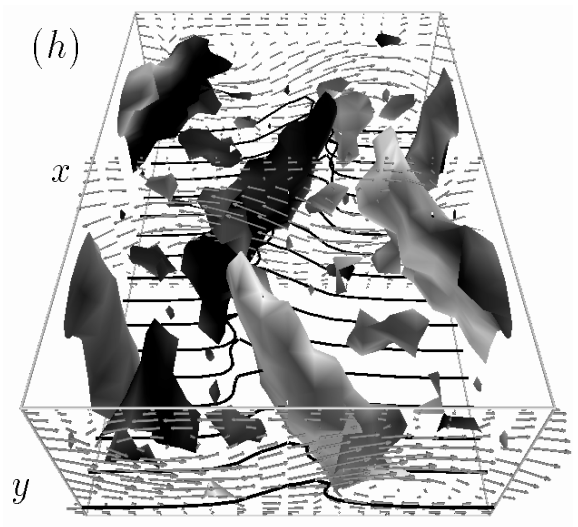

$z$

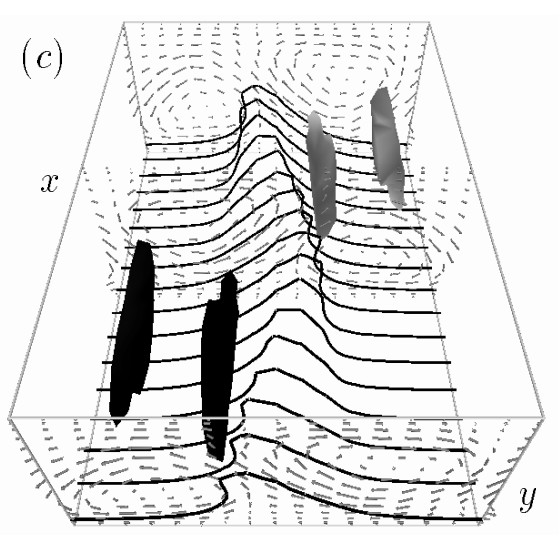

$z$

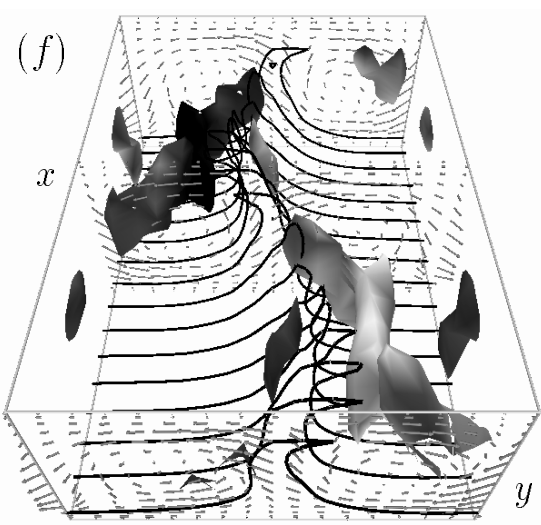

$z$

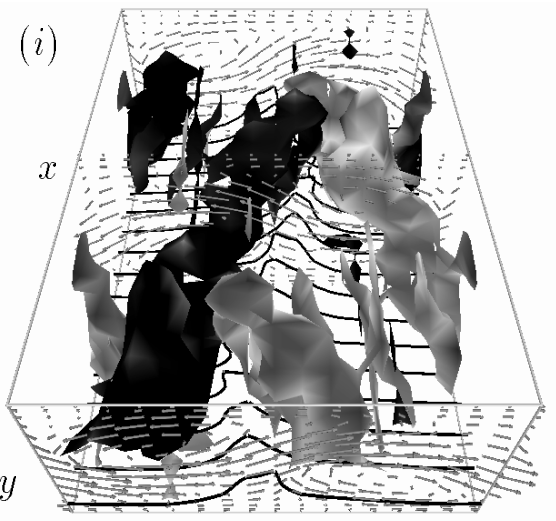

Fig. 3. A full cycle of time-periodic flow (Kawahara and Kida, 2001). Flow structures are visualised in the whole spatially periodic box $\left(L_{x} \times 2 h \times L_{z}\right)$ over one full cycle at nine times shown with open circles in Fig. 2, where panels (a) and (f) correspond respectively to the lowest and highest circles there. Time elapses from (a) to (i) by $7.2 \mathrm{~h} / \mathrm{U}$. The upper (or lower) wall moves into (or out of) the page at velocity $U$ (or $-U)$. Streamwise vortices are represented by iso-surfaces of the Laplacian of pressure, $\nabla^{2} p=0.15 \rho(U / h)^{2}$, where $\rho$ is the mass density of fluid. Brightness of the iso-surfaces of $\nabla^{2} p$ indicates the sign of the streamwise ( $x$ ) vorticity: white is positive (clockwise), black is negative (counter-clockwise). Cross-flow velocity vectors and contours of the streamwise velocity at $u=-0.3 U$ are also shown on cross-flow planes $x=$ const.

streamwise wavenumber, the zeroth-order Chebyshev polynomial, and the $2 \pi / L_{z}$ spanwise wavenumber. We have chosen the variable $\widetilde{\omega}_{y 0,0,1}$, because it represents low- and high-velocity streaks which play a crucial role in the regeneration cycle of near-wall turbulence. We here employ an iterative method to numerically obtain an unstable periodic 
orbit as a fixed point of the Poincaré map. As an initial point for the iterative method we choose a flow state that is nearly time-periodic, i.e. a point on the Poincaré plane that is mapped close to itself. We can filter such approximately periodic points from a long time integration in the turbulent state. The periodic orbit obtained in this way is drawn in Fig. 2 with a closed grey line, the period of which is $T=64.7$ $\left(T^{+}=188\right)$. Grey dots on the turbulence trajectory are much denser near the periodic orbit, implying that the turbulent state approaches it frequently. The approaches to the periodic orbit have been confirmed not only in this $(I, D)$-plane but also in other subspaces, during which the spatiotemporal structures of the turbulent flow resemble remarkably those for the periodic flow (see Fig. 3). However, the approach does not continue forever. The turbulence trajectory is destined to go away, sooner or later, from the periodic orbit. In other words, this periodic orbit should be of saddle nature.

A full cycle of the temporal evolution of spatial structure of the periodic solution is depicted in Figs. 3a-i at nine sequential phases indicated by open circles on the periodic orbit in Fig. 2. The phase of Fig. 3a corresponds to the open circle at the time of the least input and dissipation rates. We can see typical near-wall coherent structures, i.e., streaks and streamwise vortices (see Jeong et al., 1997). In Fig. 3 the clockwise (or counter-clockwise) streamwise $(x)$ vortices are visualised by the white (or black) iso-surfaces of the Laplacian of pressure, $\nabla^{2} p=0.15 \rho(U / h)^{2}$, (see also the crossflow velocity vectors) and the streamwise streaks of relatively low streamwise velocity are represented by the lifted iso-contours, $u=-0.3 U$, in the $(y, z)$-planes. The dynamics of the periodic flow is described by a cyclic sequence of events which consists of

(i) the formation and development of low-velocity streaks through the advection of streamwise velocity in the cross-flow induced by decaying streamwise vortices (Figs. 3a-d),

(ii) the bending along the streamwise direction and tilting in the spanwise $(z)$ direction of the streaks followed by the regeneration of streamwise vortices (Figs. $3 \mathrm{e}-\mathrm{g}$ ),

(iii) the breakdown of streaks and the violent development of streamwise vortices (Figs. 3h, i).

This cyclic sequence is completely consistent with a previously reported regeneration cycle (Hamilton et al., 1995).

Figure 4 compares the r.m.s. (root-mean-square) velocities for the time-periodic flow (symbols) with those for the turbulent flow (thick and thin lines), where circles and solid lines indicate the streamwise component, triangles and a dotted line the wall-normal component, and diamonds and a dashed line the spanwise component. It can be seen that the r.m.s. velocities for the time-periodic flow are in excellent agreement with those for the turbulent flow (thick lines) at the same values of $R e$ and $\left(L_{x}, L_{z}\right)$. The r.m.s. velocities for

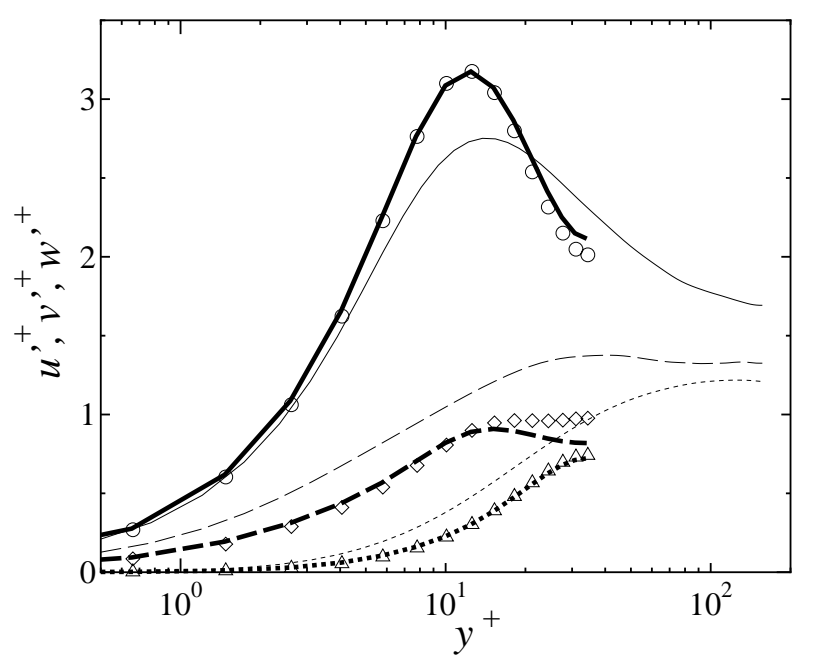

Fig. 4. Comparison of r.m.s. velocities between the time-periodic and turbulent flow. The lateral and longitudinal axes are normalised by $v / u_{\tau}$ and $u_{\tau}$, respectively. $u^{\prime+}, v^{\prime+}, w^{\prime+}$ are the streamwise, the wall-normal, and the spanwise components, respectively. The symbols represent the periodic flow. The thick lines denote the turbulent Couette flow for $R e=400$ and $\left(L_{x}^{+}, 2 h^{+}, L_{z}^{+}\right)=(188,68,128)$, while the thin lines denote the turbulent Couette flow for $R e=3000$ and $\left(L_{x}^{+}, 2 h^{+}, L_{z}^{+}\right)=(1008,320,252)$. o and solid line, the streamwise component; $\Delta$ and dotted line, the wall-normal component; $\diamond$ and dashed line, the spanwise component. The r.m.s. are computed by taking averages in time and on wall-parallel plane.

the time-periodic flow qualitatively coincide even with those for the turbulent flow (thin lines) at much higher $R e$ in the near-wall region (see also Jiménez et al., 2005).

It follows from the above results that at least in the lowReynolds-number Couette turbulence, complete spatiotemporal coherence, which is described in terms of the periodic motion, appears in incomplete shape in physical space. Since the turbulent state often approaches the periodic orbit to stay in the vicinity of it, not only the coherent structures but also the turbulence statistics are represented by the periodic motion.

Recently, for simple chaotic dynamical systems with a large number of degrees of freedom, Kawasaki and Sasa (2005) have found that there is an ensemble of unstable periodic orbits with the special property that the expectation values of macroscopic quantities can be calculated using only one unstable periodic orbit sampled from the ensemble. There should be infinitely many unstable periodic orbits embedded in Navier-Stokes turbulence. Although the systems analysed by Kawasaki and Sasa (2005) are different from the Navier-Stokes system, their results suggest that the present unstable periodic orbit might be a member of such an ensemble, and that other members of the same ensemble could also represent structures and statistics of turbulence. 


\section{Unstable periodic motion representing isotropic tur- bulence}

The unstable periodic motion discussed in Sect. 3 represents coherent structures and low-order statistics in restricted turbulent flow, i.e., near-wall turbulence in a small periodic box and at low Reynolds number. It is an interesting question whether there exists periodic motion that characterises the essence of turbulence even in larger periodic domain and at higher Reynolds numbers. In particular, if it exists, we would be interested in the relevance of periodic motion to the universal statistical laws of turbulence, e.g., the Kolmogorov energy spectrum and the logarithmic velocity profile, which are observed in fully developed turbulent flows at high Reynolds numbers. Recently, Kato and Yamada (2003) have found unstable periodic orbits in a relatively low-dimensional system for the GOY (Gledzer-Ohkitani-Yamada) shell model of turbulence. One of their periodic solutions represents not only the Kolmogorov spectrum but also the intermittency. Their findings have suggested the existence of the unstable periodic motion representing the universal statistical law. In this section we consider isotropic turbulence, which is one of the canonical forms of turbulence. We show that the NavierStokes equations for this flow allow for time-periodic solutions and we discuss periodic motion representing the Kolmogorov similarity law (van Veen et al., 2006).

In fully developed forced turbulence the energy injected in the largest-scale motion is successively transferred to the smaller-scale motion through the nonlinear interaction between different scales, and finally dissipated in the smallest scale (the Kolmogorov scale). In stationary turbulence the energy disspation rate $\epsilon$ is in balance with the energy injection rate and with the rate of energy transfer to smaller scales. At high Reynolds number $\epsilon$ is believed to be independent of the kinematic viscosity $v$. The statistical properties of the small-scale motion in high-Reynolds-number turbulence are expected to be characterised by $\epsilon$ and $\nu$. In particular, the energy spectrum normalised with $\epsilon$ and $v$ has a universal form in small scales (i.e., in the universal range), and at extremely high Reynolds numbers there appears the energy spectrum proportional to the $-5 / 3$ power of wavenumber in the inertial range that is the lower-wavenumber part of the universal range (see Davidson, 2004). This is the Kolmogorov phenomenology, and its validity has been confirmed in experiments and in numerical simulations.

In order to establish the Kolmogorov energy spectrum we should increase the Taylor-microscale Reynolds number $R e_{\lambda}$, up to more than 100 (see Kida and Murakami, 1987). It is difficult to obtain an unstable periodic solution at such a high Reynolds number even by using present-day powerful computers. Therefore we introduce the high symmetry proposed by Kida (1985). This is the largest group of discrete symmetries of the Navier-Stokes equations that allows for turbulent flows. Flows with high symmetry can be described with almost 200 times less degrees of freedom than general triply periodic flows. This allows us to compute periodic solutions.

A Fourier spectral method is used for direct numerical simulations, where the temporal integration of the NavierStokes equation is performed using the 4th-order RungeKutta-Gill scheme (Kida and Murakami, 1989). The computational domain is $2 \pi$ periodic in all the three directions. The amplitude of the low-wavenumber Fourier modes of the velocity is fixed to inject the energy in the system so that we obtain statistically stationary quasi-isotropic turbulence. Numerical computations are performed on $128^{3}$ grid points. The number of the degrees of freedom in the high-symmetric flow is about 9000 . In a weakly turbulent state at relatively low Reynolds number $R e_{\lambda} \approx 57$ we search for a recurrent state to be used as an initial guess for the Newton-Raphson iteration of periodic points of the Poincaré map. By gradually changing the kinematic viscosity $v$ the numerically obtained periodic solution is traced up to $R e_{\lambda}=67$, the highest attainable Reynolds number under the constraint of the required spatial resolution. The period of the solution is about 2.5 times longer than the characteristic timescale of the largest-scale motion in a turbulent state.

We normalise the one-dimensional longitudinal energy spectrum with $v$ and the time-average of the energy dissipation rate

$\epsilon=\frac{v}{(2 \pi)^{3}} \int_{0}^{2 \pi} \int_{0}^{2 \pi} \int_{0}^{2 \pi}|\omega|^{2} \mathrm{~d} x \mathrm{~d} y \mathrm{~d} z$.

The normalised energy spectrum is shown in Fig. 5 for the periodic solution (+) at $R e_{\lambda}=67$ and for the turbulent solution (o ) at the same conditions as that of the periodic solution. The spectra for periodic and turbulent solutions are in excellent agreement, and they are consistent with the spectra obtained by experiments (Champagne et al., 1970; • ) and by the closure theory (Kida and Goto, 1997; solid line) in the high-wavenumner range, implying that the present unstable periodic motion represents the Kolmogorov universalrange energy spectrum. Actually, it is confirmed that around $R e_{\lambda}=67$ the energy dissipation rate $\epsilon$ for the periodic motion is nearly independent of $v$ and takes almost the same value as that of the turbulence. This result also implies that the present periodic motion scales with $\epsilon$ and $v$ as in the turbulent state. As shown in Fig. 5, however, the Reynolds number, at which the periodic motion has been obtained, is not high enough to clearly show the $-5 / 3$ power spectrum observed both in the experiments and the theory.

\section{Control of Couette turbulence}

Ever since the 1990 pioneering work of Ott et al. (1990) the methods of controlling chaos have been extensively developed in low-dimensional nonlinear dynamical systems. It is known that an infinite number of unstable periodic orbits are embedded in a chaotic attractor, and in controlling chaos 


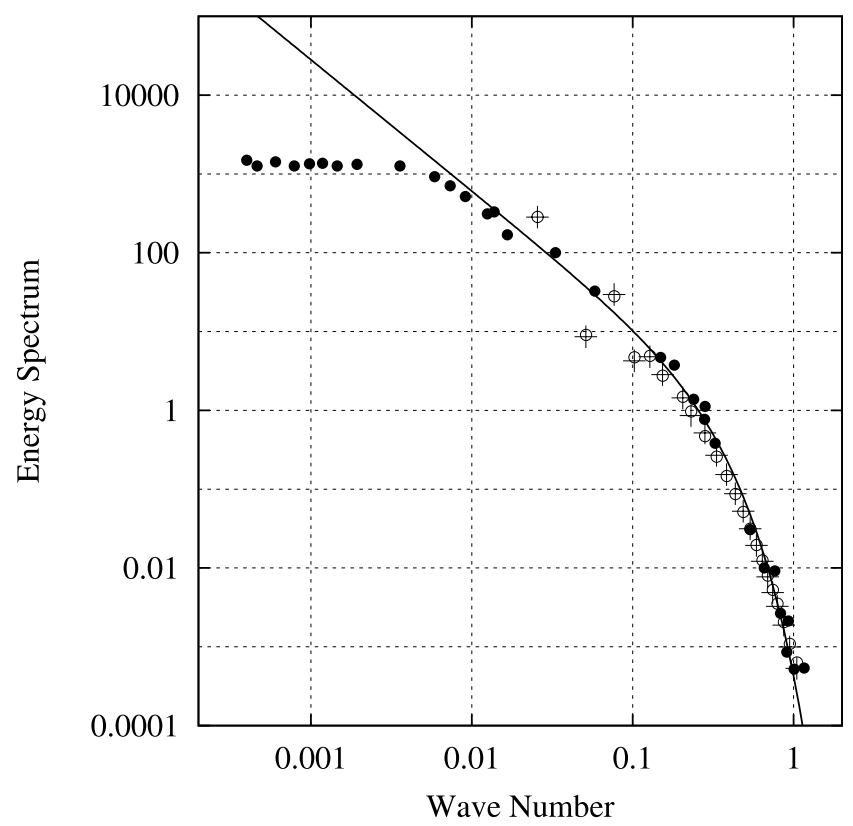

Fig. 5. One-dimensional longitudinal energy spectrum (van Veen et al., 2006). The lateral and longitudinal axes are normalised by $\left(v^{3} / \epsilon\right)^{1 / 4}$ and $\left(\epsilon \nu^{5}\right)^{1 / 4}$, respectively. + , the periodic state of highsymmetric flow at $R_{\lambda}=67$; $\circ$, the turbulent state of high-symmetric flow; $\bullet$, experimental data at $R_{\lambda}=130$ taken from Champagne et al. (1970). The solid line represents the asymptotic form at $R_{\lambda} \rightarrow \infty$ derived theoretically by the sparse direct-interaction approximation (Kida and Goto, 1997).

one of the embedded periodic orbits, which is more desirable than chaos, is stabilized by a variety of techniques (Ott et al., 1990; Pyragas, 1992). The key idea of controlling chaos is to take advantage of the sensitivity of chaotic dynamics to initial conditions or parameter values, which implies that desired states can be produced by a small change in those conditions or values. It is, however, difficult to find a nonlinear goal solution to the Navier-Stokes equation, and this is a serious obstruction to application of chaos-control strategies to fluid turbulence. In this section we discuss turbulence control strategy, i.e., a reduction in skin friction drag, inspired by investigation of the phase-space structure in the vicinity of an unstable periodic orbit (Kawahara, 2005).

The small grey closed orbit, which has been found by Kawahara and Kida (2001), with low energy input and dissipation rates $(I=D \approx 2)$ shown in Fig. 6 is an intermediary goal for control of the Couette turbulence at $R e=400$. Note that the energy input $I$ is also regarded as a skin friction normalised by that of the laminar state. As can be seen in Figs. 2 and 6 , the turbulent state occasionally approaches this quiescent periodic orbit with low skin friction drag.

Eigenvalues (or the Floquet multipliers) for the Jacobian matrix at the fixed point (the periodic solution) of the Poincaré map represent the stability characteristics of the pe-

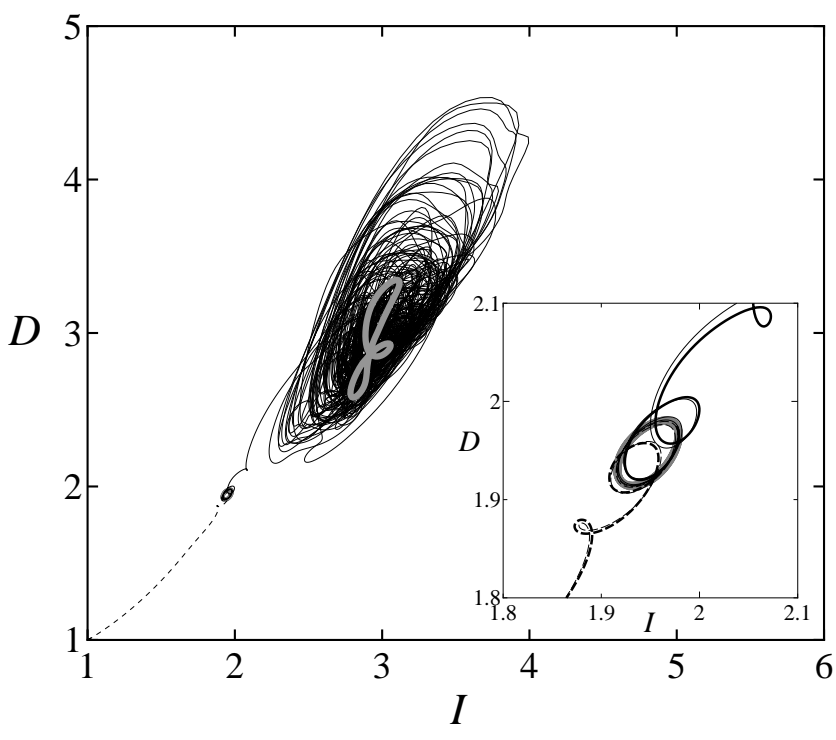

Fig. 6. The $(I, D)$-projection of two trajectories slightly perturbed in the positive (solid line) or negative (dotted line) unstable direction from the periodic orbit (Kawahara, 2005). The thick grey closed trajectory is the periodic orbit. The inset is a magnification of the two trajectories around the periodic orbit, in which two other trajectories for the flows under weak spanwise rotation $\left(2 \Omega h / U= \pm 10^{-4}\right)$ of the fluid are also shown by thick lines. The thick solid and dotted lines represent the flows under anti-parallel (positive) and parallel (negative) rotation to the mean shear vorticity, respectively.

riodic solution to infinitesimal disturbances with the same wall-parallel periods and symmetries as those of the periodic solution. It has been found that there is only one (real) unstable multiplier with modulus greater than unity, and all the others are stable. Let $\boldsymbol{e}_{u}$ denote the unit eigenvector corresponding to the unstable eigenvalue. The two trajectories shown with solid and dotted lines in Fig. 6 are the two-dimensional projection of the orbits in phase space that start respectively from the initial points $\boldsymbol{r}=\boldsymbol{r}_{f} \pm \varepsilon\left\|\boldsymbol{r}_{f}\right\| \boldsymbol{e}_{u}$ $\left(\left\|\boldsymbol{r}_{f}\right\|=0.310\right)$ on the Poincaré section, where $\boldsymbol{r}_{f}$ denotes a fixed point of the Poincaré map, and $\varepsilon=10^{-4}$. The (solid) trajectory slightly perturbed in the positive unstable direction $+\boldsymbol{e}_{u}$ from the (thick grey) periodic orbit moves to the turbulent state, while the (dotted) one perturbed in the negative unstable direction $-\boldsymbol{e}_{u}$ moves to the laminar state $(I, D)=(1,1)$. This means that the periodic orbit and its local stable manifold form a separatrix between the basin of attraction of the turbulent and the laminar states. The information on such a basin of attraction is of great importance not only for elucidation of transition mechanisms but also for controlling turbulent flows. In general it is difficult to extract a basin boundary because it should be very complicated in high-dimensional phase space. Here the discovery of the periodic orbit leads us to the extraction of the local basin boundary. In the following we shall discuss a laminarization 


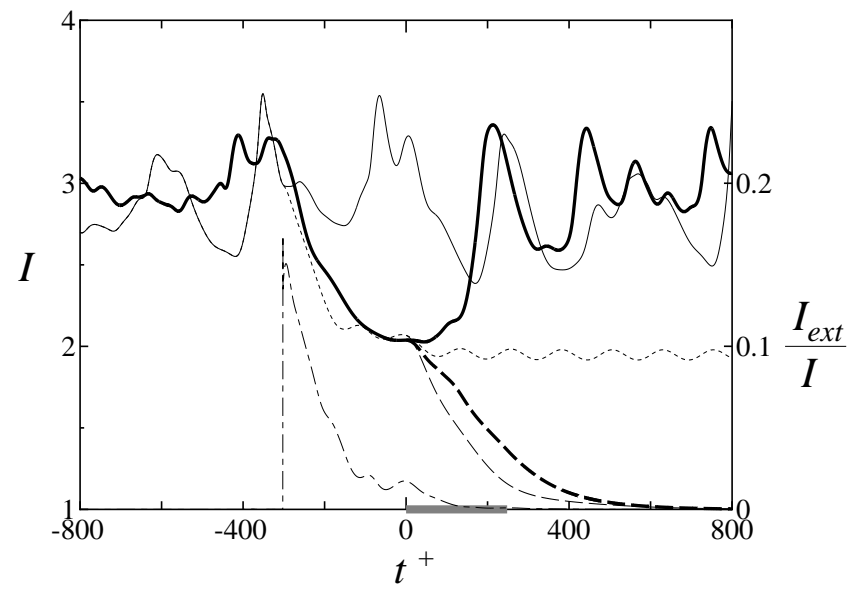

Fig. 7. Time evolution of the wall shear rate $I$ for uncontrolled and controlled flows (Kawahara, 2005). The thick and thin solid lines represent the two uncontrolled flows. One of them, shown by the thick solid line, approaches the periodic orbit naturally, and during its natural approach $\left(t^{+}=0\right)$ the rotation is turned on. The thick dashed line represents the corresponding controlled flow. The other (the thin solid line) does not approach the periodic orbit, but if Pyragas' external force with $k h / U=0.1$ is turned on at $t^{+}=-302$, the corresponding state, denoted by the thin dotted line, approaches the periodic orbit. During this forced approach $\left(t^{+}=0\right)$ the rotation is turned on, and the corresponding controlled flow is shown by the thin dashed line. The dotted-dashed line denotes the energy input $I_{\text {ext }}$ through Pyragas' forcing, normalised with $I$. The rotation is imposed for the period shown by the thick grey segment.

method for the Couette turbulence based on the knowledge of a separatrix of the basin of attraction.

It is possible to largely reduce the skin friction drag by laminarizing the turbulent flow. The laminarization is achieved by imposing small-amplitude control input for a short interval during an approach of the turbulent state to the periodic orbit so that the state point can go beyond the stable manifold (i.e., the basin boundary) toward the laminar state. For stabilization of the periodic orbit, on the other hand, the control input is determined so that the state point can fall on the stable manifold (see Ott et al., 1990). Here we introduce the rotation of the whole fluid around the spanwise axis at a constant angular velocity $\Omega$ as an example of possible control input. As shown in Fig. 6, if the rotation is parallel (or antiparallel) to the mean shear vorticity induced by the two moving plates, the state point tend to the laminar (or turbulent) state. Figure 7 shows two examples of laminarization tests. The thick solid line denotes a turbulent state without control, and the thick dashed line denotes a controlled state. It can be seen that a weak rotation, imposed during an approach of the turbulent state to the quiescent periodic orbit around $I \approx 2$, leads to the laminar state with the significantly low skin friction $I=1$. In this laminarization strategy we must wait for an approach of the turbulent state to the quiescent periodic orbit, but close approaches are not so frequent. Hence a laminarization method without such waiting is desired, which we briefly discuss in the following.

In order to make the turbulent state approach the periodic orbit at any time, we implement the external force $k \mathcal{P}\left(\boldsymbol{u}_{p}-\boldsymbol{u}\right)$ per unit mass in the Navier-Stokes equation by following Pyragas (1992), where $k$ is a (positive) constant and $\boldsymbol{u}_{p}$ and $\boldsymbol{u}$ are the velocities of the periodic flow and the flow to be controlled, respectively. The projection operator $\mathcal{P}$ provides the reconstitution of a solenoidal velocity field given only by the Fourier-Chebyshev-Fourier coefficients of the wall-normal velocity and vorticity, $\widetilde{v}_{m, l, n}$ and $\widetilde{\omega}_{y m, l, n}$, for $(m, n)=(0, \pm 1),( \pm 1,0),( \pm 1, \pm 1),( \pm 1, \mp 1)$, and $l=0,2$. Note that the number of degrees of freedom of the external force is much smaller than that of the system, $N$. The external force can globally stabilize the periodic orbit. Actually, if we turn on the forcing at any instant, the turbulent state very rapidly tends to the periodic orbit as shown in Fig. 7 (thin dotted line). After a close approach, the laminarization has been achieved in the same way as above (thin dashed line).

In order to extend this strategy to turbulent channel flows, we need the same kind of nonlinear solution to a plane Poiseuille system. Such solutions have already been found in the Poiseuille system at subcritical Reynolds numbers by Itano and Toh (2001) and Waleffe (2003). They have reported that their travelling-wave solutions are on a separatrix between the basin of attraction of turbulent and laminar states. Their solutions could be a good candidate for the base of laminarization. In the supercritical Poiseuille system, however, the laminarization demands stabilization of a laminar state.

\section{Conclusions}

In this paper we have reviewed our recent attempt at understanding and controlling turbulent flows with the aid of unstable periodic motion embedded in turbulence. Though the attempt is confined to low-Reynolds-number turbulent flows in a spatially periodic domain, it turned out that spatiotemporal coherence in such a turbulent flow is characterised in terms of unstable periodic motion, and the low-order turbulence statistics, such as r.m.s. velocities and energy spectrum, are described by the temporal average along the periodic orbit. The relevance of the unstable periodic motion to the universal statistical law, e.g. the Kolmogorov similarity law, in high-Reynolds-number turbulence has also been suggested.

Let us now recall one of the crucial subjects of turbulence research mentioned in the introduction, i.e. a quantitative explanation for what is going on in turbulent flows and how the events relate with statistical properties of turbulence. It is still difficult to extract a typical periodic motion, which represents the essence of turbulent motion, from a variety of many periodic orbits. However, if we extract such a typical motion, we might be able to understand what is going on 
in turbulent flows by fully examining exactly recurrent motion embedded in spatiotemporally complicated turbulence. By comparing the statistical properties of the periodic motion with those of turbulent motion, we might elucidate how much the coherence in turbulent flows affects the turbulence statistics. If their properties are consistent, we might be able to trace the origin of the statistical characteristics and the statistical laws of turbulent flows through the unstable periodic motion. We would like to close this paper by stating our hope and expectation that the study of unstable periodic motion will deepen our understanding of fluid turbulence and provide useful strategy for turbulence control.

Acknowledgements. G. Kawahara would like to express his thanks to M. Nagata for his encouragement and S. Toh for providing him a simulation code used in the studies in Sects. 3 and 5. This work was supported in part by a Grant-in-Aid for Scientific Research from the Japan Society for the Promotion of Science.

Edited by: A. C. L. Chian

Reviewed by: two referees

\section{References}

Auerbach, D., Cvitanović, P., Eckmann, J.-P., and Gunaratne, G. H.: Exploring chaotic motion through periodic orbits, Phys. Rev. Lett., 5, 2387-2389, 1987.

Champagne, F. H., Harris, V. G., and Corrsin, S.: Experiments on nearly homogeneous turbulent shear flow, J. Fluid Mech., 41, 81139, 1970

Christiansen, F., Cvitanović, P., and Putkaradze, V.: Spatiotemporal chaos in terms of unstable recurrent patterns, Nonlinearity, 10, 55-70, 1997.

Davidson, P. A.: Turbulence, Oxford University Press, Oxford, 2004.

Franceschini, V., Giberti, C., and Zheng, Z.: Characterization of the Lorenz attractor by unstable periodic orbits, Nonlinearity, 6 , 251-258, 1993.

Hamilton, J. M., Kim, J., and Waleffe, F.: Regeneration mechanisms of near-wall turbulence structures, J. Fluid Mech., 287, 317-348, 1995.

Holmes, P., Lumley, J. L., and Berkooz, G.: Turbulence, Coherent Structures, Dynamical Systems and Symmetry, Cambridge University Press, Cambridge, 1996.

Itano, T. and Toh, S.: The dynamics of bursting process in wall turbulence, J. Phys. Soc. Jpn., 70, 703-716, 2001.

Jeong, J., Hussain, F., Schoppa, W., and Kim, J.: Coherent structures near the wall in a turbulent channel flow, J. Fluid Mech., 332, 185-214, 1997.

Jiménez, J., Kawahara, G., Simens, M. P., Nagata, M., and Shiba, M.: Characterization of near-wall turbulence in terms of equilibrium and "bursting" solutions, Phys. Fluids, 17, 015105-1-16, 2005.
Jiménez, J. and Moin, P.: The minimal flow unit in near-wall turbulence, J. Fluid Mech., 225, 213-240, 1991.

Kato, S. and Yamada, M.: Unstable periodic solutions embedded in a shell model turbulence, Phys. Rev. E, 68, 025302-1-4, 2003.

Kawahara, G.: Laminarization of minimal plane Couette flow: Going beyond the basin of attraction of turbulence, Phys. Fluids, 17, 041702-1-4, 2005.

Kawahara, G. and Kida, S.: Periodic motion embedded in plane Couette turbulence: regeneration cycle and burst, J. Fluid Mech., 449, 291-300, 2001.

Kawasaki, M. and Sasa, S.: Statistics of unstable periodic orbits of a chaotic dynamical system with a large number of degrees of freedom, Phys. Rev. E, 72, 037202-1-4, 2005.

Kazantsev, E.: Sensitivity of the attractor of the barotropic ocean model to external influences: approach by unstable orbits, Nonlin. Processes Geophys., 8, 281-300, 2001, http://www.nonlin-processes-geophys.net/8/281/2001/.

Kida, S.: Three-dimensional periodic flows with high-symmetry, J. Phys. Soc. Jpn., 54, 2132-2136, 1985.

Kida, S. and Goto, S.: A Lagrangian direct-interaction approximation for homogeneous isotropic turbulence, J. Fluid Mech., 345, 307-345, 1997.

Kida, S. and Murakami, Y.: Kolmogorov similarity in freely decaying turbulence, Phys. Fluids, 30, 2030-2039, 1987.

Kida, S. and Murakami, Y.: Statistics of velocity gradients in turbulence at moderate Reynolds numbers, Fluid Dyn. Res., 4, 347370, 1989.

Kim, J., Moin, P., and Moser, R.: Turbulence statistics in fully developed channel flow at low Reynolds number, J. Fluid Mech. 177, 133-166, 1987.

Nagata, M.: Three-dimensional finite-amplitude solutions in plane Couette flow: bifurcation from infinity, J. Fluid Mech., 217, 519527, 1990.

Ott, E., Grebogi, C., and Yorke, J. A.: Controlling chaos, Phys. Rev. Lett., 64, 1196-1199, 1990.

Pyragas, K.: Continuous control of chaos by self-controlling feedback, Phys. Lett. A, 40, 421-428, 1992.

Reynolds, O.: An experimental investigation of the circumstances which determine whether the motion of water shall be direct or sinuous, and of the law of resistance in parallel channels, Phil. Trans. R. Soc. London, 174, 935-982, 1883.

van Veen, L., Kida, S., and Kawahara, G.: Periodic motion representing isotropic turbulence, Fluid Dyn. Res., 38, 19-46, 2006.

Waleffe, F.: Homotopy of exact coherent structures in plane shear flows, Phys. Fluids, 15, 1517-1534, 2003.

Zgliczyński, P.: Rigorous numerics for dissipative partial differential equations II. Periodic orbit for the Kuramoto-Sivashinsky PDE - a computer-assisted proof, Found. Comput. Math., 4, 157-185, 2004.

Zoldi, S. M. and Greenside, H. S.: Spatially localized unstable periodic orbits of a high-dimensional chaotic system, Phys. Rev. E, 57, R2511-R2514, 1998. 